\title{
Design Considerations and Establishment of a Low Frequency Hydrophone Calibration Setup using the Principle of Vibrating Water Column
}

\author{
A. Malarkodi, G. Latha and PSSR Sridhar \\ National Institute of Ocean Technology, Pallikaranai, Chennai, India. \\ Niels V. Bøgholm \\ Brüel \& Kjar S\&V Measurements A/S, Skodsborgvej 3072850 Narum, Denmark
}

(Received 22 June 2015; accepted: 5 October 2016)

This paper describes the design and implementation of a low frequency hydrophone calibration system, using a vibrating water column. The hydrophone to be calibrated is immersed in the water column and the position of the transducer is kept constant while a hydrodynamic pressure field is generated in the water column by means of a shaker (similar to what is described in IEC 60565). F. Schloss et al. used a vibrating water column for hydrophone calibration in the frequency range from $10 \mathrm{~Hz}$ to $700 \mathrm{~Hz} .{ }^{1}$ An interlaboratory comparison calibration was carried out by the Russian Metrological Institute of Technical Physics and Radio Engineering (VNIIFTRI) and Hangzhou Applied Acoustics Research Institute (HAARI) in the frequency range from $250 \mathrm{~Hz}$ to $1 \mathrm{kHz}$. $^{2}$ The dimensions of the test vessel are important for deciding the frequency range of operation. Simulations were carried out for the selection of vessel material and dimension. To overcome limitations in the frequency range caused by resonance in the water column, the principle of operation was modified from absolute calibration to calibration by comparison. By using a single cylindrical vessel, the frequency range is extended to cover frequencies from less than $100 \mathrm{~Hz}$ to $1 \mathrm{kHz}$. The calibrated reference hydrophone type Bruel \& Kjaer (B\&K) 8104 is used in this calibration. Some results obtained from the use of the calibration system are also presented in this paper.

\section{INTRODUCTION}

There is an increasing requirement to perform measurements of sound in the ocean for both environmental monitoring and assessment. Most of the sound sources of environmental concern, including marine species, emit much of their energy in the $20 \mathrm{~Hz}$ to $1 \mathrm{kHz}$ frequency range. Traceable calibrations of the hydrophones used are required to support these measurements. However, free field calibration of hydrophones in laboratory test tanks is not possible at such frequencies (due to limited tank dimensions), necessitating the use of calibration methods such as the one described herein. This calibration is fundamental for accurate measurements and to estimate the characteristics of hydrophones. The most significant parameter in specifying hydrophone performance is its free field sensitivity $\left(M_{H}\right)$ expressed as a function of frequency. $M_{H}$ can be expressed as a quotient of the voltage and sound pressure: ${ }^{1}$

$$
M_{H}=\frac{v}{p}
$$

where $v$ is the electrical output voltage from the hydrophone and $p$ is the acoustic pressure acting on the hydrophone.

In general, the sensitivity can be measured using comparison and reciprocity calibration methods in a free field environment (e.g. acoustical water tanks, ponds, and lakes). Free field methods are limited with respect to the lowest frequency of operation. If the calibration is carried out with a tone burst signal, the received signals may be contaminated by transients. This is due to the resonant behaviour of the transducers, which depend on the $Q$ factor. Further, the duration of the tone burst must be limited in order to avoid interference between the direct signal and signal reflections from the water surface and tank walls.
As the calibration frequency is decreased, the number of cycles in the useable time window is reduced. This effect defines a lower limiting frequency for measurements made using tone burst signals.

As an alternative to free field calibration in an acoustical water tank, a number of different methods for low frequency calibration of hydrophones exist. These include calibration by piezoelectric compensation, ${ }^{3}$ acoustic coupler reciprocity calibration ${ }^{4}$ and calibration with a pistophone. ${ }^{5}$ In the piezoelectric compensation method, the hydrophone is calibrated in an enclosed small water-filled chamber. This method, which works only for frequencies with a wavelength sufficiently larger than the largest dimension of the chamber, ${ }^{3}$ is useful for calibration in the $1 \mathrm{~Hz}$ to $5 \mathrm{kHz}$ frequency range. For our required frequencies, more than one chamber is required in order to cover the full frequency range. Acoustic coupler reciprocity calibration is an absolute calibration method using the reciprocity principle. ${ }^{4}$ Though laboratory standard hydrophones may be calibrated with the highest accuracy, the coupler is not a good option to calibrate hydrophones that are large or to calibrate hydrophones near the resonance frequency. In a pistophone, a continuous sound pressure is generated in an enclosed small air-filled chamber by a vibrating piston..$^{5}$ This technique may not be used at frequencies greater than the upper limiting frequency because of non-uniform pressure in the chamber.

The benefit of using water versus air is that, due to different propagation velocities in water and air, the wavelength in water is much longer than that in air; therefore the useable frequency range is expanded by the relation between the two velocities. An absolute pressure calibration of the hydrophone can also be performed at low frequencies by immersing the hydrophone in 
a column of liquid and oscillating the liquid column vertically. ${ }^{6}$ This can result in large pressure oscillations and eliminates any possible response of the hydrophone to its own acceleration. The hydrophone sensitivity is calculated from the acoustic pressure measured at a particular depth. Various limitations on the procedure, such as those due to fluid motion around the hydrophone and wave effects in the liquid column, are discussed. ${ }^{7}$ Strasberg et al. explained several advantages calibrating the pressure gradient hydrophones in a cylinder of liquid, wherein they are subjected to axial vibration (as opposed to horizontal orientation of the cylinder). ${ }^{4}$ In the vibrating water column method, the hydrophone under calibration is immersed in a water column; the position of the transducer is kept constant while the fluid column is moved sinusoidally. Schloss et al. used the vibrating water column method for the calibration of hydrophones, wherein the cylinder is placed over the vibrating shaker and half the cylinder is filled with water. ${ }^{8}$ The vibrating column method has also been used for interlaboratory comparison calibrations in the frequency range $250 \mathrm{~Hz}$ to $1 \mathrm{kHz}$ by the VNIIFTRI and HAARI, China. ${ }^{2}$ Here the up and down motion of the cylinder is driven by the vibrating shaker and the hydrophone element is exposed to an oscillating pressure fluctuation.

Bauer et al. developed the calibrator for pressure gradient hydrophone by using a very rigid tank and testing in the frequency range $3 \mathrm{~Hz}$ to $2.5 \mathrm{kHz}$. ${ }^{9}$ Here two calibrated pressure hydrophones are lowered to the same depth as a pressure gradient hydrophone, then the system is set into axial oscillation. The difference in pressure is then measured by differential amplifier.

All of the above low frequency calibration techniques, either in air or water, are pressure calibration methods. While free field sensitivity is the significant performance parameter for hydrophones, at such low frequencies the pressure sensitivity and free field sensitivity are equivalent. Hence the vibrating water column based hydrophone calibration setup has been taken up.

The objective of the current work was to establish a system for calibration of hydrophones in the frequency range $100 \mathrm{~Hz}$ to $1 \mathrm{kHz}$ (calibration at higher frequencies is performed in the acoustic tank by free field calibration method). Matjaz Prek studied the sound propagation and resonance conditions for different water filled pipe materials. ${ }^{10}$ An experimental study of sound propagation in liquid-filled ducts has established the utility of wall materials for sound attenuation. ${ }^{11}$ In a vertical vibrating water column, the hydrophone is exposed to two forces. Firstly, the gravitational force due to the small displacement of the water column. Secondly it is exposed to an hydrodynamic force according to Newton's second law $F=m * a$ (where $m$ is the mass of the vibrating water column above the hydrophone and $a$ is its acceleration).

Above a certain frequency the gravitational forces can be neglected, and the acoustic pressure inside the water column can be written as:

$$
p=\rho x h \omega^{2}
$$

where $\rho$ is the density of the liquid, $h$ is the height of the water column above the acoustical center of the hydrophone, $\omega$ is the angular frequency, and $x$ is the peak amplitude of vibration.

The sensitivity of the hydrophone can be obtained from the calculated pressure at the depth of the hydrophone and the measured open circuit voltage. ${ }^{12}$ The sensitivity of the hydrophone $(M)$ is found as the ratio of the open circuit voltage $\left(V_{O}\right)$ and the pressure $(p)$. The absolute calibration suffers from limitations due to the resonant effects of the setup. To overcome the influence of the resonant phenomena, a reference hydrophone is introduced to measure the actual acoustic pressure.

\section{METHODOLOGY}

The vibrating water column method requires a test vessel (often cylindrical), hydrophone fixtures, a vibration shaker, and instrumentation for signal generation and signal acquisition. It is very important that the design of the hydrophone fixtures is such that no external vibrations are picked up by the hydrophone. The diameter of the test vessel must be large and sufficient water must be around the hydrophone in order to avoid the influence of the boundary effects. To choose the optimum test vessel, a simulation was carried out with different materials and wall thicknesses.

\subsection{Modelling and Simulation}

The acoustic pressure of the water field and the vibration of the structure around the water field are modelled and simulated using the software COMSOL Multiphysics. The pressure acoustics tool available in COMSOL Multiphysics is used to model the isolated pressure within the water. ${ }^{13}$ Initially, calibration using a water column as given in IEC 60565 was simulated. Then the behaviour of the pressure field in the water for different dimensions and structure materials were modeled and simulated. Finally the responses of the hydrophones with different dimensions and different water column levels were studied.

The acoustical impedance of water is high; hence, the shell around the water column must be of such a nature that it can be considered rigid. ${ }^{14,15}$ If the shell is not sufficiently rigid it will influence the water pressure and vice versa. Several materials such as Aluminum, Steel and, PVC (all with different thickness) have been considered for the calibration vessel. It was noticed that the frequency response of a PVC vessel has several peaks, which makes it unsuitable for calibration purposes. The resonance for all other materials is above $1 \mathrm{kHz}$. Based on the simulation, it was found that an aluminium cylinder with $20 \mathrm{~mm}$ wall thickness, because of its reduced weight when compared with a steel cylinder with the same dimensions, would be the most feasible choice for the calibration setup. The dimensions of the vessel were chosen to be small enough to keep the pressure field uniform throughout the vessel. During the study, different dimensions of the hydrophones were simulated. The simulations showed that the resonances can be shifted up in the frequency if the length of the water column is shorter. The shortest water column length is considered the most desirable in order to shift resonant frequency as high as possible. It is however expected that the hydrophone is positioned at the center of the water column in order to avoid the influence of boundary effects. As described in IEC 60565, the vibrating water column method is an absolute calibration with a very limited frequency range. The comparison calibration principle used here allows an extension of the frequency range up to $1 \mathrm{kHz}$ or more.

\section{DESIGN CONSIDERATIONS}

The establishment of the setup, including mechanical structures and instrumentation, was carried out by Brel \& Kjr $(\mathrm{B} \& \mathrm{~K})$, Denmark. The main elements of the calibration system 


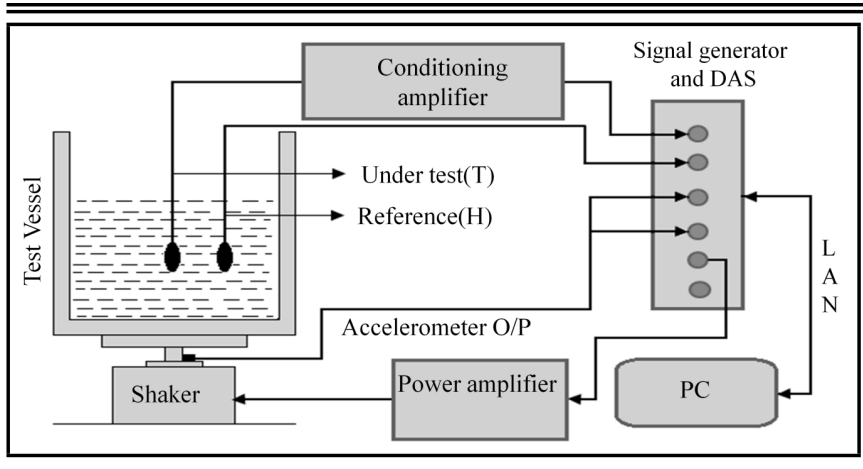

Figure 1. Measurement Schematic.

include a cylindrical test vessel, vibration shaker, signal generator, power amplifier, data-acquisition system, accelerometer, and hydrophone fixture arrangement. The developed system is intended for the calibration of hydrophones in the frequency range of $100 \mathrm{~Hz}$ to $1 \mathrm{kHz}$ utilizing the vibrating water column method. This method uses an open column of liquid sized such that the wavelength is larger than the length of the column. The cylindrical vessel with rigid walls that contains the liquid column is driven at the bottom by the shaker arrangement. Some of the challenges overcome by this design were: the selection of the vibration shaker (including static deflection of the shaker head due to the static load of the test vessel with water), the hydrodynamic force rating, standing waves of the water column (including mode shapes), and finally the selection of the vessel material with the required dimensions. The upper frequency of the calibration range depends on the dimensions of the device under test and the total height of the water column. The upper limit of the useful frequency range is smaller than a quarter of the wavelength of the sound in the liquid. The test vessel is an aluminium tube with $0.3 \mathrm{~m}$ diameter and $0.02 \mathrm{~m}$ wall thickness. If the payload is being carried by the shaker itself, the static payload from the water and vessel will result in a large deflection of the shaker head. To avoid this static deflection, the walls of the test vessel are made to rest on the foundation via a trunnion arrangement and, further, the static load of the water column is carried by the pressurized air.

Only the bottom of the test vessel is driven by the shaker arrangement which oscillates the entire column of liquid vertically. An arrangement of O-rings provides the sealing between the walls of the test vessel and the vibrating bottom. A triaxial piezoelectric accelerometer is used for the measurement of the axial and transverse vibrations of the shaker with respect to the pressure created in the water column.

\section{MEASUREMENT METHOD}

The implemented system uses calibration by comparison and is a modification of an absolute method described in IEC 60565. ${ }^{1}$ A reference hydrophone $(\mathrm{H})$ and the unit under test (T) are placed in a hydrodynamic pressure field (water), with the acoustical centers at the midpoint of the water column with the accuracy of $\pm 2 \mathrm{~mm}$. The sensitivity of the hydrophone under test is determined from the sensitivity of a known reference standard. The excitation signal is a sine/random signal with bandwidth limitation. The auto-spectra for the frequency response function (FRF) is measured by means of $1 / 24$ octave $\mathrm{CPB}$ filters in this method.

The voltage sensitivity of unknown hydrophone SVT can be written as:

$$
S V_{T}=S V_{H} *\left[U_{T} / U_{H}\right]
$$

$$
M_{T}=M_{H}+20 \log \left[U_{T} / U_{H}\right] ;
$$

where:

$S V_{H} \quad-\quad$ Voltage sensitivity of reference hydrophone;

$U_{T} \quad-\quad$ Output voltage of hydrophone T;

$U_{H} \quad-\quad$ Output voltage of hydrophone $\mathrm{H}$;

$M_{H} \quad-\quad$ Sensitivity level of reference transducer $(\mathrm{dB}$ re $\mathrm{V} / \mu \mathrm{Pa})$;

$M_{T} \quad-\quad$ Sensitivity level of hydrophone $T$ (dB re $\mathrm{V} / \mu \mathrm{Pa})$.

The values of $M_{H}$ for the reference hydrophone type, B\&K 8104, are taken from the National Physical Laboratory (NPL), UK calibration certificate, and at 1/24 octave frequencies considered by interpolation where needed.

The response as calculated above needs to be corrected for (1) losses due to the capacitive load of the reference and device under test (2) filter and gain testings in the preamplifier, amd (3) any narrow frequency range resonance phenomena in the vessel. All these factors are considered by a number of corrections as shown in the complete formula below.

$$
M_{T}=M_{H}+H_{1} G_{N}+C_{c}+C_{L}+C_{F}+C_{V} ;
$$

where:
$M_{T} \quad-\quad$ Receiving sensitivity of the hydrophone under test as a function of frequency;
$M_{H} \quad$ - Reference transducer sensitivity as a function of frequency;
$H_{1} \quad$ - Transfer function between under test hydrophone and the reference hydrophone $\left(U_{T} / U_{H}\right)$;
$G_{N} \quad-\quad$ Correction factor for preamplifier gain-if used;
$C_{C}-$ Correction for the capacitive load effect;
$C_{L} \quad-\quad$ Load error, caused by the resistive load for low frequencies;
$C_{F} \quad-\quad$ Correction for the filter response (wherever relevant);
$C_{V} \quad-\quad$ Vessel correction, a small correction for reso- nances in the test vessel. This is small correc- tion for standing waves and it is frequency and hydrophone type dependent.

Each calibration may have its own individual set of corrections. In general, corrections due to the gain of the preamplifier $\left(G_{N}\right.$ in $\left.\mathrm{dB}\right)$ are the same for all the frequencies of operation. However, for better accuracy one can calibrate the amplifier to characterize the gain at each frequency point. The hydrophone sensitivity and frequency response must be stated as open circuit values at the end of the hydrophone cable (i.e., the value measured without any load). Hence, whenever the hydrophone is connected with the rest of the circuit, which is attempting to measure the output voltage, the capacitance due to the hydrophone and the extension cable must be corrected for. This correction is called $\mathrm{Cc}$ and its value depends on the capacitance of the load and the input capacitance of the measuring channel.

If both the transducers are considered identical, or nearly identical, the correction will be zero or close to zero. For hydrophones with a relatively low capacitance $(C<4 \mathrm{nF})$, the effect of $C_{L}$ should be corrected for. However, since both the unknown and the reference have the same loading effect, the transfer function (i.e., the ratio) is less influenced than a single channel. In that case the vessel dimension and other circumstances cause small glitches in the response. A correction $\left(C_{V}\right)$ can be used to correct these phenomena. 


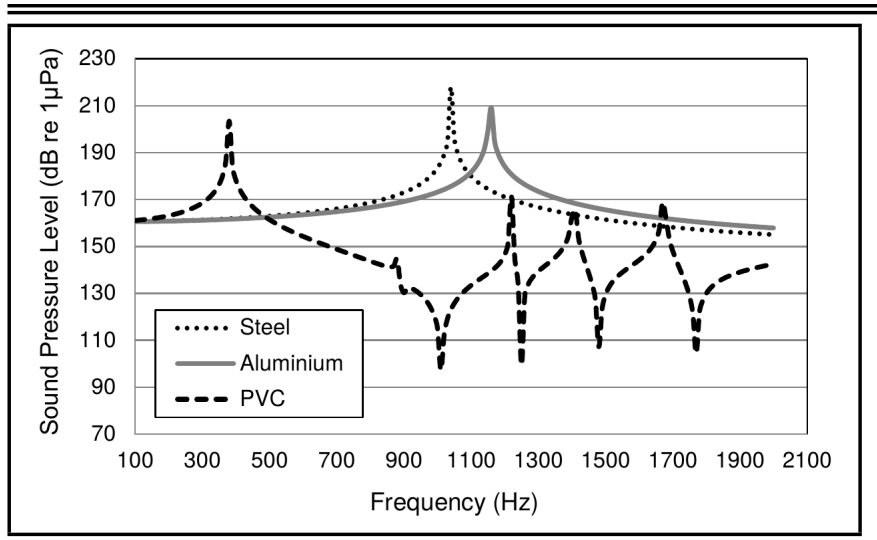

Figure 2. Comparison of the responses for different cylinder materials.

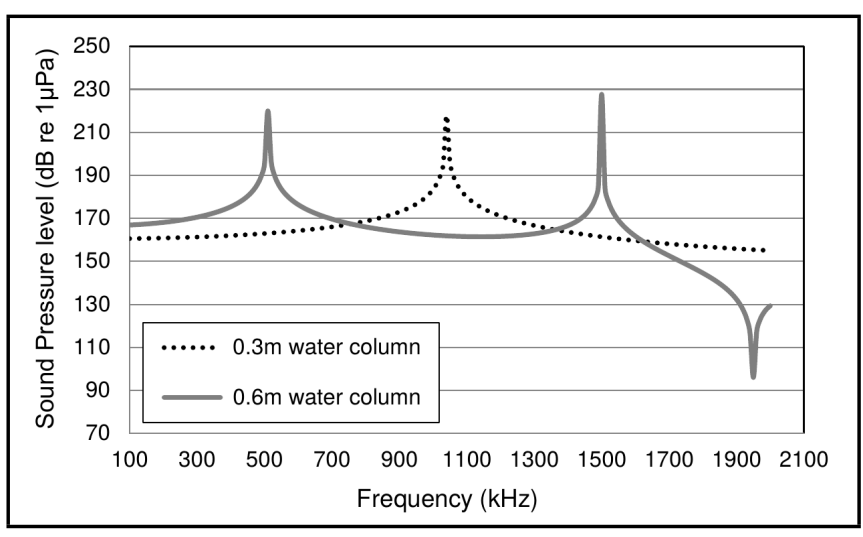

Figure 3. Comparison of the response for different water column heights.

\section{RESULTS AND DISCUSSION}

COMSOL simulation demonstrated that the vibrating water column principle described in IEC 60565 can be extended to frequencies above $c /(16 * L)$ where $c$ is the speed of sound in water and $L$ is the length of the vibrating water column. The frequency range where this resonance gives a peak can be estimated based on dimensions and material constants. This is because the wave speed can change according to the shell and the exact water length. Figure 2 illustrates the simulation of the pressure in a water column with a height of $0.3 \mathrm{~m}$ for steel, aluminium and PVC cylinder materials of thickness $0.02 \mathrm{~m}$. The graph for PVC shows that the first resonance occurs approximately at $350 \mathrm{~Hz}$ and multiple peaks are observed. Hence, PVC has not been considered for further modelling. Though the steel cylinder performance is equally good compared with the aluminium cylinder, steel has not been taken up due to its weight.

The simulation is also carried out for an $0.3 \mathrm{~m}$ diameter aluminium cylinder of $0.02 \mathrm{~m}$ wall thickness with different water column heights. If the water height is increased there is a shift in the resonance frequencies. As the water column increases the first resonant moves towards the low frequency. The response for water column heights of $0.3 \mathrm{~m}$ and $0.6 \mathrm{~m}$ are shown in Fig. 3.

Figure 4 shows the test vessel with the shaker beneath it. Figures 5 and 6 show the measured receiving sensitivity for hydrophones B\&K type 8103 and Cetacean type C55, respectively, in the frequency range of $100 \mathrm{~Hz}$ to $1 \mathrm{kHz}$. The values are stated in $\mathrm{dB}$ re $\mathrm{V} / \mu \mathrm{Pa}$.

The reference hydrophone type B\&K $8104(\mathrm{H})$ used in this system was calibrated at NPL, UK and the calibration uncer-

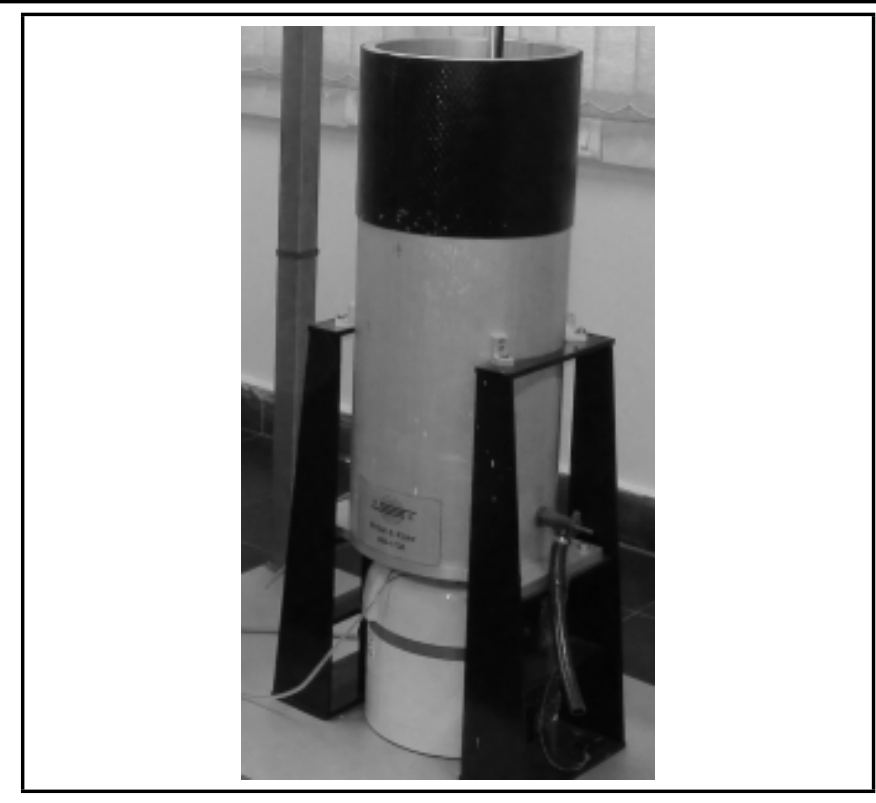

Figure 4. Hydrophone calibration system.

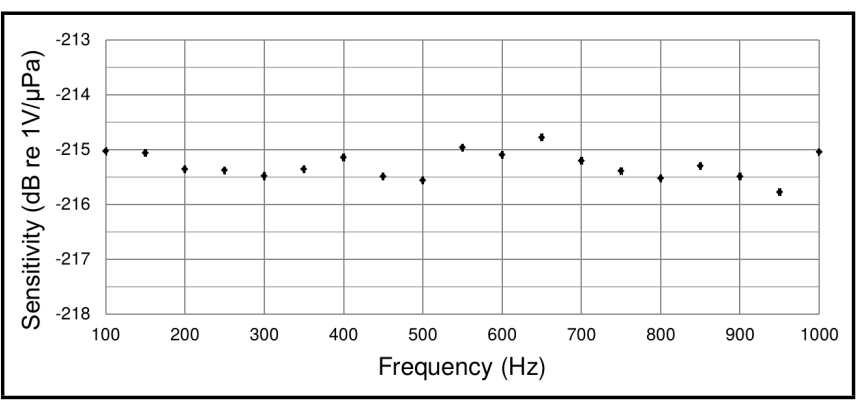

Figure 5. Sensitivity of hydrophone Bruel \& Kjaer type 8103.

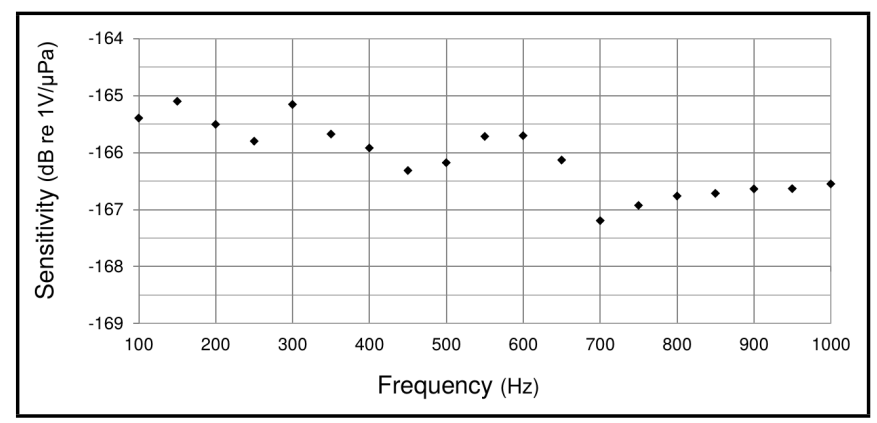

Figure 6. Sensitivity of hydrophone CETACEAN type C55.

tainty is directly included as one element in the calibration uncertainty. In NPL, UK, the low frequency range $25 \mathrm{~Hz}$ to $250 \mathrm{~Hz}$ was calibrated by the technique of air pistophone and the upper frequency range up to $1 \mathrm{kHz}$ is calibrated by three transducer spherical wave reciprocity. The uncertainty budget is made in accordance with the Guide to the Expression of Uncertainty in Measurement (GUM). ${ }^{16}$ The sensitivity of the unknown hydrophone is calculated by Eq. (5). The major uncertainty is due to the reference hydrophone.

However, all major sources are considered for computing overall measurement uncertainty. The sources of uncertainty are uncertainty due to reference hydrophone, uncertainty for capacitive load correction, vessel correction, uncertainty from filter (if used), and accuracy of the data acquisition system(see Table 1). These uncertainty values are all dependent 


\begin{tabular}{l}
\hline \hline Table 1. List of sources of uncertainty and its values in $\mathrm{dB}(k=2)$. \\
$\qquad$\begin{tabular}{||l|l|l|}
\hline & Sources of uncertainty & $\begin{array}{l}\text { Standard } \\
\text { uncertainty } \\
(k=2)\end{array}$ \\
\hline 1 & Uncertainty for reference hydrophone & $\pm 0.6 \mathrm{~dB}$ \\
\hline 2 & Uncertainty for capacitive load correction & $\pm 0.2 \mathrm{~dB}$ \\
\hline 3 & $\begin{array}{l}\text { Capacitive load correction } \\
\text { (depends on the hydrophone) }\end{array}$ & $\pm 0.2 \mathrm{~dB}$ \\
\hline 4 & Correction for resonances in the vessel & $\pm 0.2 \mathrm{~dB}$ \\
\hline 5 & Uncertainty from filter (if used) & $\pm 0.05 \mathrm{~dB}$ \\
\hline 6 & Accuracy of the data acquisition system & $\pm 0.03 \mathrm{~dB}$ \\
\hline
\end{tabular}
\end{tabular}

on the hydrophone and frequency. All relevant uncertainties are summed using the root of summed squares for calculating combined uncertainty. Then the expanded uncertainty is computed from combined uncertainty (i.e., combined uncertainty is multiplied with the coverage factor $k=2$ to a confidence level of $95 \%$ ). The uncertainty table is given as below.

\section{CONCLUSIONS}

Simulations using COMSOL were carried out for different water column heights and different vessel materials — such as steel, aluminium, and PVC - of different thicknesses. The simulation results demonstrated that the vibrating water column principle explained in IEC 60565 can be applied for calibration of hydrophones with certain limitations (due to the resonance phenomena of the water column). To overcome these limitations the principle of comparison calibration with a reference hydrophone was used in order to extend the frequency range. After successful simulation, an aluminium vessel with $0.3 \mathrm{~m}$ diameter and $0.02 \mathrm{~m}$ wall thickness was manufactured with the trunnion arrangement to avoid static load deflection. The calibration procedure was prepared by considering all the correction factors, such as the capacitive load effect of the hydrophone cable, resistive load, correction for resonances in the test vessel, and correction for filter. The random signal with the bandwidth of $1.6 \mathrm{kHz}$ was given to the shaker. The frequency response of the hydrophone under test and reference hydrophone are acquired by the data acquisition system. The sensitivity of the under test hydrophone was estimated from transfer function $\left(H_{1}\right)$ along with corrections. Here the major sources of uncertainty are due to the reference hydrophone uncertainty. Though the calibration frequency range is $1 \mathrm{kHz}$, the overall uncertainty can be minimized by increasing the wall thickness of the vessel and decreasing the water column height for smaller dimensional hydrophones, whereby the upper frequency may be extended.

Further investigations and potential future improvements are given below:

- Closer investigation of the useable frequency range in the absolute mode.

- Use a set of very high input impedance hydrophone preamplifiers in order to avoid some of the present corrections; this can simplify the operation of the system.

- Investigation of the system performance at lower frequencies, down to around $30 \mathrm{~Hz}$ is expected to be possible.

\section{ACKNOWLEDGEMENTS}

The authors would like to express their sincere thanks to the director, National Institute of Ocean Technology for the support given for developing the vibrating water column based calibration system at their Acoustic Test Facility.

\section{REFERENCES}

1 Bobber, R.J. Underwater Electroacoustic Measurements, Peninsula Publication, Los Altos, (1990).

2 Chen Yi., Isaev, A.E., Yuebing, W., Enyakov, A.M., Teng, F., and Matveev, A.N. Comparison of hydrophone calibrations in the frequency range $250 \mathrm{~Hz}$ to $200 \mathrm{kHz}$, Coomet Pilot Comparison 473/RU-a/09, 48 Tech. Suppl. 09004, Metrologia, (2011).

3 Golenkov, A.N., and Nekrich, S.F. Features of the calibration of hydrophones in the nonuniform field of measuring chambers. Measurement Techniques, 37, 1301, (1994).

4 Mcmahon, G.A. Coupler-Reciprocity system for hydrophone calibration at high pressures. J. Acoust. Soc. Am., 36, 2311, (1964).

5 Lipscomb, L.W.I. An instrument semi-automatic acoustic calibration system and attachment thereto. Proceedings of Institute of Acoustics, 6, 113, (1985).

6 Strasberg, M. and Schloss, F. Calibration of pressuregradient transducers in an oscillating liquid column, $J$. Acoust. Soc. Am., 54, 553, (1973).

7 Schloss, F. and Strasberg, M. Hydrophone calibration in a vibrating column of Liquid, J. Acoust. Soc. Am., 34, 958, (1962).

8 Schloss, F., County, A., and Strasberg, M. Patent No: 3224246, Low frequency hydrophone calibration, (1963)

9 Baurer, B., Louis, A., and Schumann, J. Wide-Range Calibration System for Pressure-Gradient Hydrophones, $J$. Acoust. Soc. Am., 51, 1717, (1972).

10 Prek, M., Experimental Determination of the Speed of Sound in Viscoelastic Pipes International Journal of Acoustics and Vibration, 5 (3), 146-150, (2000).

11 Horne, M.P. and Hansen R. J., Sound propagation in a pipe containing a liquid of comparable acoustic impedance, $J$. Acoust. Soc. Am., 71 (6), 1400-1405, (1982).

12 IEC 60565:2006. Underwater acousticsHydrophones Calibration in the frequency range $0.01 \mathrm{~Hz}$ to $1 \mathrm{MHz}$, International Electrotechnical Commission, Geneva, (2006).

13 Comsol 5.0 - Acoustics Module Users Guide.

14 Kinsler L.E., Frey A.R., Coppens A. B., and Sanders J.V., Fundamentals of Acoustics, Wiley \& Sons, New York, (2000).

15 Jacobsen, F., Propagation of Sound Waves in Ducts, DTUNote: 31260, Danish Technical University, (2011).

16 JCGM 100-2008, Evaluation of measurement data Guide to the expression of uncertainty in measurement, BIPM, IEC, IFCC, ILAC, ISO, IUPAC, IUPAP and OIML (2008). 